\title{
3D CLASSIFICATION OPTIMIZATION OF AIRBORNE LIDAR DATA BY PARAMETERS SELECTION
}

Baha Eddine Aissou $^{1}$, Aichouche Belhadj Aissa ${ }^{1}$

${ }^{1}$ Image Processing and Radiation Laboratory, University of Sciences and Technology Houari Boumediene, USTHB, Bp 32 El Alia, Bab Ezzouar Alger, 16111, Algeria.

Correspondence to: Baha Eddine Aissou (bahae.aissou@gmail.com)

https://doi.org/10.31490/9788024845050-1

\begin{abstract}
Airborne Laser Scanning (ALS) point cloud segmentation and classification are very important and attractive tasks that still interest many scientists. In this paper, 3D classification optimization method is applied for of airborne LIDAR point cloud, covering an urban area with a low density. Our main contribution is to solve classifier and kernel hyperparameters tuning issue. Typically, choice of parameters is done empirically. In almost all classification studies, no details are given for the classifier parameters choice, which is very important. The parameters of the classifier have a direct impact on the classification results. Finding hyper-parameters leads to optimal classification results. In order to determinate the hyper-parameters, authors propose to apply in the context of LiDAR data, a method proposed in the literature called parameters selection to deduce the best parameters, in order to optimize the classifications results. The results shown that parameters selection does not systematically lead to hyper-parameters, but can be used as first stage, then a fine search can be performed around the obtained interval.
\end{abstract}

Keywords: LiDAR airborne, Support Vector Machine, RBF kernel, parameters selection.

\section{EXTENDED ABSTRACT}

\section{Introduction}

Light Detection and Ranging (LiDAR) is an active remote sensing technology used for several applications. Airborne LiDAR has become an indisputable tool for the acquisition of highly accurate and dense point cloud. A classification of Airborne Laser Scanning (ALS) point cloud is very important task that still interest many scientists. Classification approaches can be categorized in point-based and segment-based classification. For point-based classification, the attributes is calculated for each point. While with segment-based method, a prior segmentation of point cloud is required, followed by attributes computing for the resulting segments [1]. Support Vector Machine (SVM), based on kernels, is one of the most used statistical learning algorithm for LiDAR data [2]. Only a few training samples, called support vectors, are required. SVM has shown its potential to cope with uncertainty in data caused by the noise and fluctuation, and its computationally efficient compared to several other methods [3]. Such properties are particularly suited for remote sensing classification problems and explains their large adoption [4]. 
The article addresses the problem of classification parameters tuning. The choice of classifier and kernel parameters is pertinent and has a significant impact on the classification results. Little information is provided in the articles regarding the choice criterion of classification parameters. The tuning of classification parameters is usually made empirically after several testing. The parameters of the classifier have a direct impact on the classification results. Finding hyper-parameters leads to optimal classification results.

Authors propose to apply parameters selection method proposed in [7], in the context of LiDAR data to optimize the classifications results. Hyper-parameters are deducted by performing grid search on SVM classifier parameters and 5 -fold cross validation.

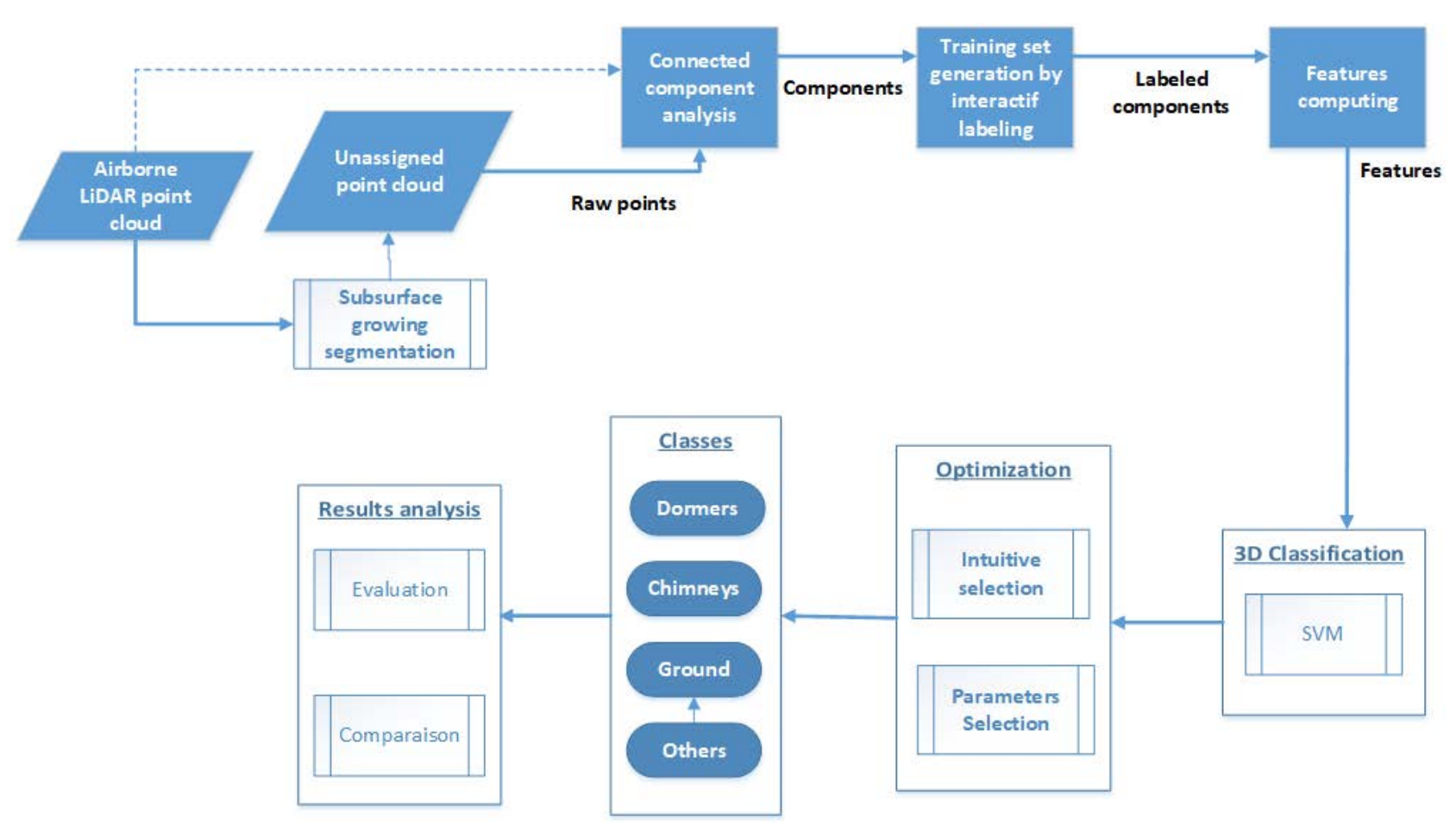

Fig.1: the flowchart of the present study.

\section{Methodology}

In this paper, 3D classification optimization of ALS LiDAR point cloud is proposed. The targeted classes concern roof-building elements, often omitted by researchers, due to their small size and shape variety. Firstly, sub-surface segmentation (SSG) method is applied on point cloud as pretreatment stage, which permit to discard buildings roofs segments. Further details regarding sub-surface growing segmentation method can be found in [5]. Secondly, the unassigned raw point cloud SSG, is clustered using connected component analysis method in order to group the points into distinct objects. The applied clustering method is known for image processing and was successfully used for LiDAR data [4]. Connected component analysis only requires a neighborhood distance as an initial parameter. Thirdly, twelve geometric-based attributes are computed for each object. The components are projected over the orthophoto of the studied area, which is used as ground truth. Then, the component are labelled one by one, 1412 components containing 25695 
points. Fig. 2 shows the selected areas in the orthophoto for classifier training. The flowchart of this study is shown in Fig. 1.

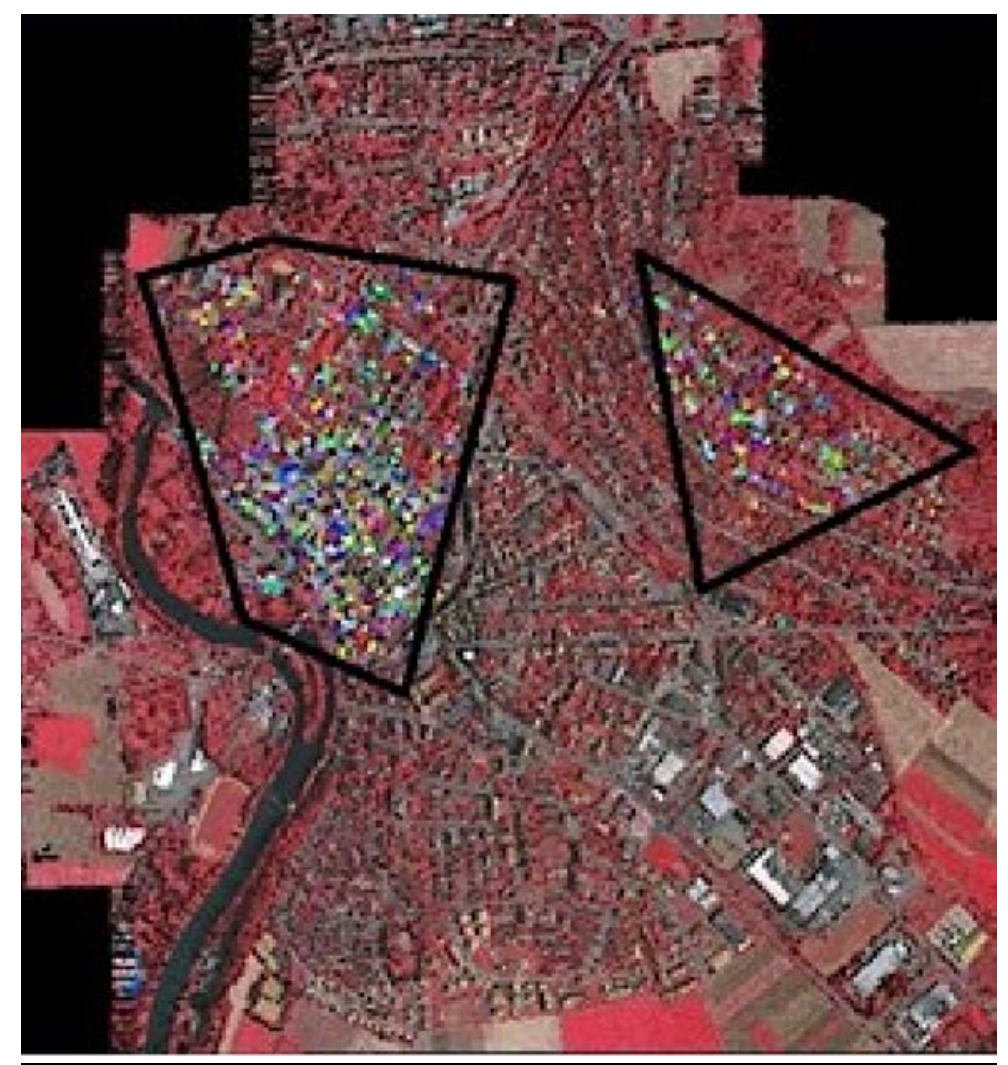

Fig. 2: the selected areas used labelled for training the classifier

\section{Data Results and Discussion}

The exploited LiDAR point cloud is provided by the German Society for Photogrammetry, Remote Sensing and Geoinformation [8]. The ALS data used is characterized by a low density (4-6 points $/ \mathrm{m}^{2}$ ), and is covering an urban area, located in residential parts of the city Vaihingen in southern Germany. Two areas are selected in the orthophoto (in Fig.2) and an interactive labeling of the underlying components is performed to form the training data set. Then, the labelled set is randomly split into training (70\%) and test subsets (30\%). The classes considered are ground, others and two classes belonging to the roof building (dormers and chimneys), making four classes. Finally, the components are subjected to a SVMs classifier. The Radial Basic Function RBF Kernel is used. The choice of Gaussian kernel is motivated firstly by the fact that the kernel was successfully adopted in several articles [4] [6]. Secondly, the use of a more sophisticated kernel does not involve an enhancement of the results. Thirdly, the small number of the required parameters $(C$ and $\sigma)$ to decrease the computation time. In addition to overall accuracy $(\mathrm{OA})$, the performance measurements of classification used are the recall, precision, F-score and area under the curve (AUC) score.

In order to optimize the classification rates, the parameter selection is inspected. It consists to find the kernel and classifier parameters ( $\mathrm{C}$ and $\sigma$ ) leading to best overall accuracy using grid search and v-fold cross validation. The 5 -fold cross validation accuracy is computed several times while varying $C$ in $\left[2^{-15}, 2^{-13}, \ldots 2^{-1}, 2^{1}, 2^{3}, \ldots 2^{15}\right]$ and $\sigma$ in 
$\left[2^{-15}, 2^{-13}, \ldots 2^{-1}, 2^{1}, 2^{3}\right]$. The pairwise $(C, \sigma)$ giving the higher cross validation accuracy is retained for the final classification process. More details about parameters selection can be found in [7]. With the same way, a finer grid search can be conducted in a limited interval around the selected parameters in previous stage, in order to enhance further the overall accuracy. Supposing for example the parameters selection resulted to $C=2^{5}$ and $\sigma=2^{-3}$ as initial best parameter, then the fine grid search is just the application of grid search by:

varying $C$ and $\sigma$ in the restricted intervals $\left\{2^{4}, 2^{4.5}, 2^{4.75}, \ldots 2^{5}, 2^{5.25}, 2^{5.5}, 2^{6}\right\}$ and $\left\{2^{-4}, 2^{-3.75}, 2^{-3.5}, 2^{-3}, 2^{-3.5}, 2^{-2.5}, 2^{-2}\right\}$ respectively.

Table1: performance results of SVM classifier using parameter selection $(C=2048, \sigma=8)$

\begin{tabular}{lllll}
\hline Classes & classes & Precision & Recall & F1-score \\
\hline & Dormer & 0.83 & 0.86 & 0.85 \\
\cline { 2 - 5 } & Chimney & 0.89 & 0.98 & 0.93 \\
\cline { 2 - 5 } & Ground & 0.88 & 0.96 & 0.92 \\
\cline { 2 - 5 } & Others & 0.85 & 0.93 & 0.89 \\
\hline
\end{tabular}

\begin{tabular}{cc}
\hline OA & $85.22 \%$ \\
\hline AUC & 0.93 \\
\hline
\end{tabular}

Table2: performance results of SVM classifier using intuitive search of parameters $(\mathrm{C}=$ 1092, $\sigma=5.5)$

\begin{tabular}{cllll}
\hline Classes & classes & Precision & Recall & F1-score \\
\hline & Dormer & 0.83 & 0.89 & 0.86 \\
\cline { 2 - 5 } & Chimney & 0.89 & 0.99 & 0.94 \\
\cline { 2 - 5 } & Ground & 0.96 & 0.98 & 0.97 \\
\cline { 2 - 3 } & Others & 0.86 & 0.97 & 0.91 \\
\hline OA & $\mathbf{8 8 . 3 1 \%}$ & & & \\
\hline AUC & $\mathbf{0 . 9 6}$ & & & \\
\hline
\end{tabular}

As can be shown in table 1 , parameters selection has led to ( $C$ and $\sigma$ ) equal to $(2048,8)$ respectively. The achieved results should be the highest. However, the table 2 shows that the deducted parameters results, $(C$ and $\sigma$ ) equal to $(1092,5.5)$ outperform those obtained by parameters selection. Intuitive search, which consists to apply parameters selection, than flowed by empiric search around the obtained interval, achieved to the optimal OA and F1score.

\section{Conclusion}

The present study demonstrated that for LiDAR point cloud, parameters selection proposed in [7], [9] does not systematically lead to hyper-parameters as claimed. Even though parameters selection has been successfully exploited for different remote sensing data [10, 11]. The particularity of the considered classes may influence the classification optimization. 
Parameters selected empirically gave better results. We demonstrated that hyperparameters can be determined by intuitive parameters selection, Which consists to orient the search in a restricted interval of $(C$ and $\sigma)$ after parameter selection step.

\section{References:}

[1] G. Vosselman, M. Coenen, and F. Rottensteiner, "Contextual segment-based classification of airborne laser scanner data," ISPRS J. Photogramm. Remote Sens., vol. 128, pp. 354-371, 2017.

[2] C. Mallet, F. Bretar, M. Roux, C. Heipke, and U. Soergel, "Relevance assessment of full-waveform lidar data for urban area classification," ISPRS J. Photogramm. Remote Sens., vol. 66, no. 6, pp. S71-S84, 2011.

[3] M. Satari, "Recognition of Dormers from lidar data using support vector machine," in International Geoscience and Remote Sensing Symposium (IGARSS), 2012, pp. 6005-6008.

[4] Aissou, B., and A. Belhadj Aissa. 2020. "An Adapted Connected Component Labeling For Clustering Non-Planar Objects From Airborne Lidar Point Cloud." International Archives of the Photogrammetry, Remote Sensing and Spatial Information Sciences - ISPRS Archives 43 (B2): 191-95. https://doi.org/10.5194/isprs-archives-XLIII-B2-2020-191-2020.

[5] M. Kada and A. Wichmann, "SUB-SURFACE GROWING and BOUNDARY GENERALIZATION for 3D BUILDING RECONSTRUCTION," in ISPRS Annals of the Photogrammetry, Remote Sensing and Spatial Information Sciences, 2012, vol. 1, no. September, pp. 233-238.

[6] T. Kavzoglu and I. Colkesen, "A kernel functions analysis for support vector machines for land cover classification,” Int. J. Appl. Earth Obs. Geoinf., vol. 11, no. 5, pp. 352-359, 2009.

[7] Chih-Wei Hsu, Chih-Chung Chang, Chih-Wei Hsu, Chih-Chung Chang, C.-J. Lin, and C.-J. L. ChihWei Hsu, Chih-Chung Chang, Chih-Wei Hsu, Chih-Chung Chang, and C.-J. Lin, "A Practical Guide to Support Vector Classification," BJU Int., vol. 101, no. 1, pp. 1396-1400, 2008.

[8] M. Cramer, "The DGPF-Test on Digital Airborne Camera Evaluation - Overview and Test Design," Photogramm. - Fernerkundung - Geoinf., vol. 2010, no. 2, pp. 73-82, 2010.

[9] Kuo, Bor Chen, Hsin Hua Ho, Cheng Hsuan Li, Chih Cheng Hung, and Jin Shiuh Taur.. "A Kernel-Based Feature Selection Method for SVM with RBF Kernel for Hyperspectral Image Classification." IEEE Journal of Selected Topics in Applied Earth Observations and Remote Sensing 7 (1): 317-26., 2014.

[10] M. Carranza-garcía, J. García-gutiérrez, and J. C. Riquelme, "A Framework for Evaluating Land Use and Land Cover Classification Using Convolutional Neural Networks," 2019.

[11] A. A. Matkan, M. Hajeb, and S. Sadeghian, "Road Extraction from Lidar Data Using Support Vector Machine Classification," Photogramm. Eng. Remote Sens., pp. 409-422, 2014. 